\title{
Reproducibility of ultrasound attenuation imaging for the noninvasive evaluation of hepatic steatosis
}

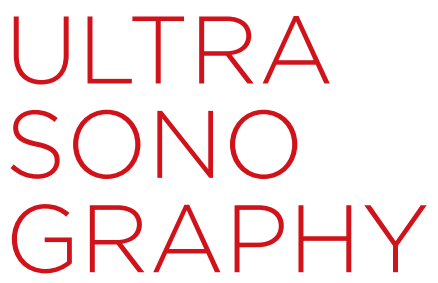

\author{
Jeongin Yoo ${ }^{1}$, Jeong Min Lee ${ }^{1,2}$, ljin Joo ${ }^{1,2}$, Dong Ho Lee ${ }^{1,2}$, Jeong Hee Yoon ${ }^{1,2}$, \\ Hyo-Jin Kang', Su Joa Ahn ${ }^{3}$ \\ ${ }^{1}$ Department of Radiology, Seoul National University Hospital, Seoul; ${ }^{2}$ Institute of Radiation \\ Medicine, Seoul National University Medical Research Center, Seoul; ${ }^{3}$ Department of \\ Radiology, Gachon University Gil Medical Center, Gachon University College of Medicine, \\ Incheon, Korea
}

Purpose: The purpose of this study was to evaluate the intra-observer reproducibility of ultrasound attenuation imaging (ATI) for the noninvasive assessment of hepatic steatosis in patients with suspected hepatic steatosis and the inter-observer reproducibility in asymptomatic volunteers.

Methods: This prospective study was approved by our institutional review board and informed consent was obtained from all patients. In group 1, composed of patients with suspected hepatic steatosis ( $n=143$ ), one abdominal radiologist performed gray-scale ultrasonography and two sessions of ATI. In group 2, composed of healthy volunteers $(n=18)$, three independent sessions of ATI were performed by three abdominal radiologists. The visual degree of hepatic steatosis in all study subjects was graded on a 4-point scale by two independent reviewers using gray-scale ultrasonography. Thereafter, the attenuation coefficient (AC) was correlated with the degree of hepatic steatosis using Spearman rank correlation analysis. Intra-class correlation coefficients (ICCS) were used to assess the intra-observer (group 1) and inter-observer reproducibility (group 2) of ATI measurements.

Results: For the intra-observer reproducibility of ATI, the ICC was 0.929 ( $95 \%$ confidence interval $[\mathrm{CI}], 0.901$ to 0.949$)$, and the coefficient of variation was $7.1 \%$. Inter-observer reproducibility of ATI measurements showed an ICC of $0.792(95 \% \mathrm{Cl}, 0.549$ to 0.916$)$. The AC showed a significant correlation with the visual grade of hepatic steatosis for both reviewers (rho, 0.780 and 0.695; $\mathrm{P}<0.001$, respectively).

Conclusion: ATI showed high intra- and inter-observer reproducibility in the assessment of hepatic steatosis.

Keywords: Ultrasonography; Non-alcoholic fatty liver disease; Obesity

\section{ORIGINAL ARTICLE}

https://doi.org/10.14366/usg. 19034 pISSN: 2288-5919 • elSSN: 2288-5943 Ultrasonography 2020;39:121-129

Received: June 15, 2019

Revised: August 7, 2019

Accepted: August 12, 2019

Correspondence to: Jeong Min Lee, MD, Department of Radiology and Institute of Radiation Medicine, Seoul National University College of Medicine, 103 Daehak-ro, Jongno-gu, Seoul 03080, Korea

Tel. +82-2-2072-3154

Fax. +82-2-743-6385

E-mail: jmlshy2000@gmail.com

This is an Open Access article distributed under the terms of the Creative Commons Attribution NonCommercial License (http://creativecommons.org/ licenses/by-nc/4.0/) which permits unrestricted noncommercial use distribution, and reproduction in any medium, provided the original work is properly cited.

Copyright @ 2020 Korean Society of Ultrasound in Medicine (KSUM)

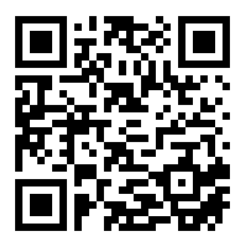

How to cite this article:

Yoo J, Lee JM, Joo I, Lee DH, Yoon JH, Kang $\mathrm{HJ}$ et al. Reproducibility of ultrasound attenuation imaging for the noninvasive evaluation of hepatic steatosis. Ultrasonography. 2020 Apr;39(2):121-129. 


\section{Introduction}

Hepatic steatosis is defined as triglyceride content exceeding $5 \%$ in hepatocytes [1]. The most common causes of hepatic steatosis are obesity and its complications, especially type 2 diabetes and hypertriglyceridemia [2]. Indeed, nonalcoholic fatty liver disease (NAFLD) is the most common cause of liver disease worldwide, with a reported global prevalence of $25.2 \%$ [3]. NAFLD can progress into a more advanced stage of disease such as cirrhosis or nonalcoholic steatohepatitis (NASH) [3,4]. In addition, hepatic steatosis is a common histopathological feature of chronic hepatitis B (CHB) [5] and chronic hepatic C (CHC) infections [6]. Moreover, the presence of steatosis has been reported to be associated with faster fibrosis progression or significant fibrosis burden in patients with CHC or CHB [7-10]. Therefore, an easy-to-use tool that can diagnose hepatic steatosis via simple, noninvasive evaluation of liver fat content in different clinical settings would be beneficial for the identification of asymptomatic high-risk patients and the evaluation of response to therapy or disease progression [11].

At present, the gold standard for diagnosing hepatic steatosis is a liver biopsy. However, liver biopsy has clear disadvantages of morbidity owing to invasiveness, cost, and inaccuracy due to sampling variation $[12,13]$. In accordance with clinical needs, quantitative imaging-based approaches using either magnetic resonance (MR) or ultrasonography (US) techniques have emerged as noninvasive tools for steatosis quantification [14-17]. Previous studies have demonstrated that quantitative US parameters such as the attenuation coefficient (AC) and backscatter coefficient may be more accurate than conventional US for predicting the hepatic steatosis grade in patients with NAFLD $[18,19]$. AC is a measure of US energy loss in tissue that provides a numerical parameter analogous to the obscuration of tissue structures assessed qualitatively using B-mode images [20]. Recently, 2-dimensional (2D) attenuation imaging (ATI) has been developed and equipped on a clinical US platform (Canon Medial Systems, Tochigi, Japan), which allows tissue characterization by providing the $A C$ and color mapping [21]. ATI is expected to have an advantage from the perspective of real-time measurements and large sample volumes [22]. However, for ATI to be used more widely, a high probability of obtaining actual applicable measurements and repeatability are warranted. To the best of our knowledge, no study has evaluated the intra-observer or inter-observer agreement of AC measured using the real-time $2 \mathrm{D}$ ATI technique.

The purpose of this prospective study, therefore, was to evaluate the reproducibility of real-time $2 \mathrm{D} \mathrm{ATI}$ measurements for the noninvasive assessment of hepatic steatosis.

\section{Materials and Methods}

Two separate prospective studies (one for intra-observer reproducibility of ATI in patients with suspected hepatic steatosis [group 1] and the other for inter-observer reproducibility of ATI in volunteers (group 2]) were approved by our institutional review board. Informed consent was obtained from all enrolled subjects.

\section{Patients}

In group 1, 157 patients who met the following eligibility criteria from January to May 2018 were enrolled: (1) older than 18 years of age; (2) clinically suspected hepatic steatosis with or without liver function test abnormalities; and (3) scheduled for initial or follow-up liver US examinations at our radiology department. Fourteen patients were excluded according to the following criteria: (1) moderate, heavy, or binge alcohol consumption, defined as three drinks or more per day on average for men and two or more drinks per day on average for women $(n=6)$; $(2)$ inability to hold their breath for more than 3 seconds during the US examination $(n=4)$; (3) patients who had undergone right hepatectomy $(n=2)$; and (4) patients who had received locoregional treatments for liver malignancies $(n=2)$. Laboratory tests, including liver biochemistry and body mass index (BMI) measurements, were performed in patients when clinically indicated at the discretion of the referring physicians, on the same day. Finally, 143 patients constituted the study population.

For group 2, we separately enrolled 18 asymptomatic adult volunteers for evaluation of the inter-observer reproducibility of ATI. All volunteers had no history of chronic viral or non-viral hepatitis such as alcoholic or non-alcoholic steatohepatitis, or autoimmune hepatitis. Laboratory tests, including liver biochemistry and measurements of BMI, were obtained from all subjects on the same day.

\section{Gray-Scale US and ATI Measurements Group 1}

One board-certified radiologist (J.M.L.) with 20 years of experience in abdominal imaging performed conventional gray-scale US examinations using a clinical US system (Aplio i900, Canon Medical Systems) and a wide-band convex i8CX1 multifrequency probe. Patients were requested to fast for at least 6 hours before the examination. Sonograms were obtained from patients in the supine or decubitus position and in the subcostal, transverse, oblique, sagittal, and intercostal planes. The sonograms were stored for evaluation of the presence or absence of the known US features of fatty liver, including increased echogenicity, impaired visualization of the diaphragm line and intra-hepatic portal vein wall, and decreased visualization of deep liver structures [23]. Additionally, the visual 
degree of hepatic steatosis was scored later by two independent reviewers on gray-scale US as follows: 0 (absent), 1 (mild), 2 (moderate), and 3 (severe). Mild steatosis was defined as increased liver echogenicity compared with the right renal cortex [24]. Moderate steatosis was indicated by liver echogenicity greater than that of the right renal cortex with obscured periportal echogenicity (Fig. 1A). Severe steatosis was determined by increased liver echogenicity obscuring diaphragmatic echogenicity. Additionally, the skin-to-capsule distance was measured as the shortest distance between the transducer and the Glisson capsule.

The same radiologist who acquired gray-scale US also performed additional ATI measurements, which provided color mapping of the degree of ultrasound attenuation using the same clinical US system (Aplio i900, Canon Medical Systems) in all patients. Two sessions of ATI measurements were performed by the radiologist, with an interval of 2-5 minutes and a positional change between sessions. According to the vendors' recommendation, the radiologist attempted to obtain at least six ATI color maps with an $R^{2}$ value $\geq 0.8$. An approximately $3 \times 4-\mathrm{cm}$ region of interest (ROI) overlaid on the B-mode image was placed in the right lobe of the liver at the intercostal space while the patient held his or her breath (Fig. 1B). The ROI box was placed in a relatively homogeneous region of the right lobe of the liver at least $2 \mathrm{~cm}$ below the liver capsule, while avoiding areas with focal fat sparing or deposition and large vessels. With a single button pressed by the operator for freezing a B-mode image, the level of US attenuation was color-coded and displayed in the ROI. Areas with significant errors of attenuation calculation, such as vascular structures or areas with reverberation artifacts, were excluded from the ATI map.

In principle, on gray-scale imaging, an ultrasound beam is focusdependent, so that the highest image resolution and maximum signal intensity are acquired at the focal zone while the signal intensity profile is adjusted and transformed to the simple signal intensity profile, which reflects the attenuation caused by scattering and absorption in ATI. The beam profile is then obtained by averaging the signal from the yellow ROI, and $A C$ is automatically quantified. The US system automatically displays the $A C$ and the results of the quality of linear fitting for the reliability of data $\left(R^{2}\right.$ value) to optimize the accuracy of ROI placement. The $R^{2}$ value can be interpreted as follows: $<0.8$, poor; 0.81-0.90, good; $>0.9$, excellent. Since $A C$ values with an $R^{2}<0.8$ were considered to be unreliable and were excluded from the analysis, the radiologist performed ATI measurements until each session contained six AC measurements with an $R^{2} \geq 0.8$. The ATI values in each set were averaged for statistical analysis.

\section{Group 2}

Three board-certified radiologists (H.J.K., S.J.A., J.M.L.) with at least 6 years of experience in abdominal imaging performed ATI measurements using an Aplio i900 (Canon Medical Systems). All

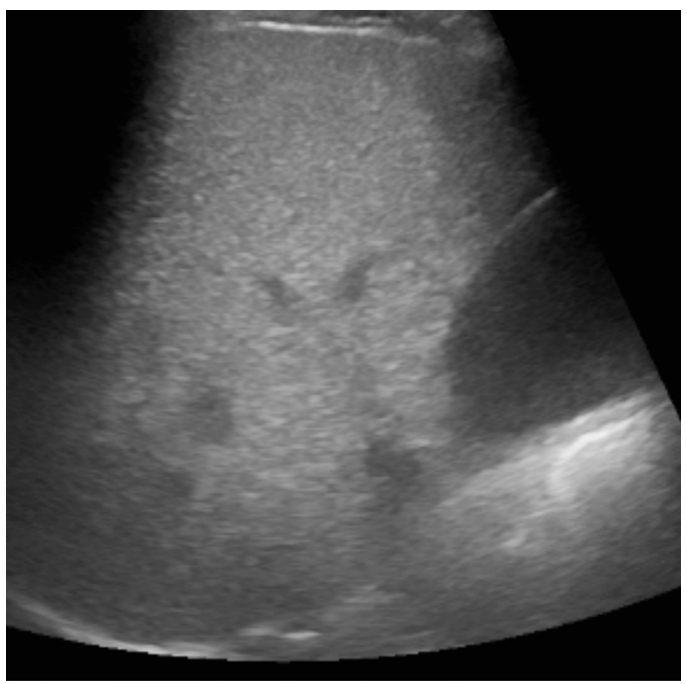

A

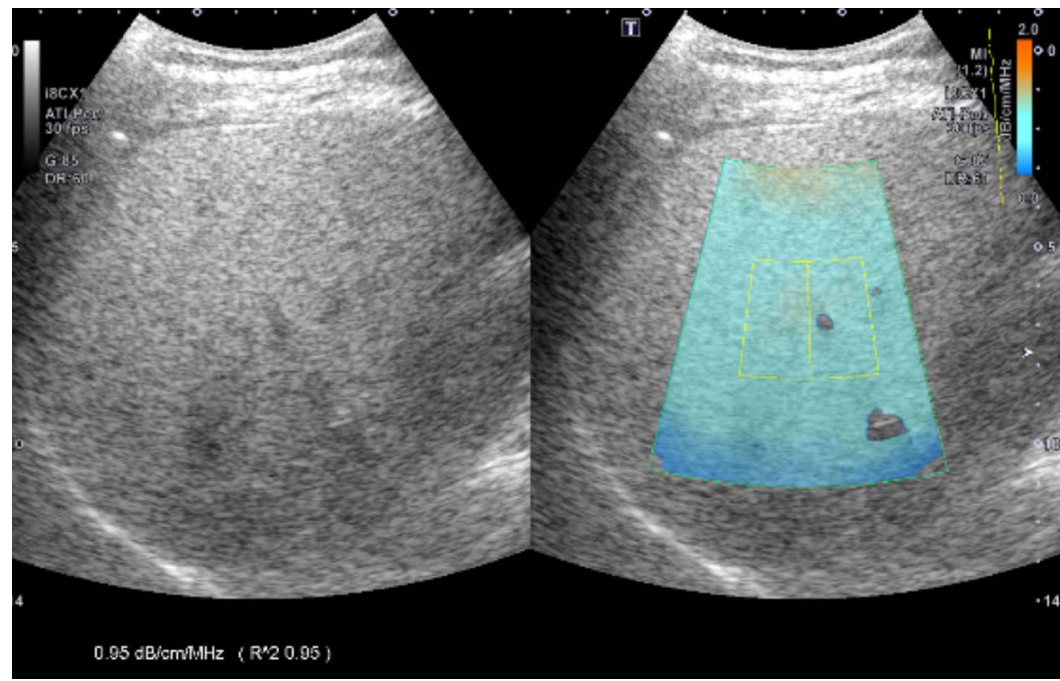

B

Fig. 1. Gray-scale ultrasound and attenuation imaging (ATI) in a 48-year-old man with hepatic steatosis.

A. Gray-scale ultrasound imaging shows increased liver echogenicity with obscured periportal echogenicity and preserved diaphragmatic echogenicity. Both reviewers 1 and 2 assessed the degree of steatosis as 2 (moderate). B. ATI was performed in the right lobe of the liver through an intercostal scan. The level of attenuation was color-coded and displayed in the region of interest (ROI), excluding vascular structures. The ultrasound system automatically displays the attenuation coefficient $(\mathrm{dB} / \mathrm{cm} / \mathrm{MHz})$ and the coefficient of determination $\left(\mathrm{R}^{2}\right.$ value) to optimize the accuracy of ROI placement. 
volunteers had fasted for at least 6 hours before the examination. Each radiologist measured the $A C$ at least 6 times in each volunteer. The method of measurement was the same as that for group 1 as described above. Two radiologists who performed a visual assessment of steatosis in group 2 also assessed the degree of hepatic steatosis, independently and retrospectively.

\section{Statistical Analysis}

Correlations between the mean $\mathrm{AC}$ and visual grades of steatosis determined by the two reviewers were assessed using Spearman rank correlation coefficients. The AC was compared between the patients with different visual grades using the Jonckheere-Terpstra and Mann-Whitney $U$ tests. Comparison of visual grading by two reviewers was analyzed using the Wilcoxon signed-rank test, and inter-observer agreement between the two reviewers for visual assessment was evaluated using linear-weighted kappa statistics. The kappa results were interpreted as follows: values $<0.2$ as poor, $0.21-0.40$ as fair, $0.41-0.60$ as moderate, $0.61-0.80$ as good, and $0.81-1.00$ as very good agreement. The intra-observer (group 1) and inter-observer reproducibility (group 2) of ATI measurements and intra-observer reproducibility according to BMI were assessed using intra-class correlation coefficients (ICCS) and the coefficient of variation (CoV). ICC estimates and their 95\% confidence intervals (Cls) were calculated based on a mean-rating, absolute-agreement, 2-way mixed-effects model. Based on the $95 \% \mathrm{Cl}$ of the ICC estimate, values less than 0.5 , between 0.5 and 0.75 , between 0.75 and 0.9 , and greater than 0.90 were considered indicative of poor, moderate, good, and excellent reliability, respectively [25]. All statistical analyses were performed using IBM SPSS Statistics for Windows version 20.0 (IBM Corp., Armonk, NY, USA) and MedCalc Statistical Software version 15.2 (MedCalc Software, Ostend, Belgium; http://www.medcalc.org; 2015). A P-value of $<0.05$ was considered indicative of statistical significance.

\section{Results}

\section{Patient Characteristics Group 1}

Among 143 patients (76 men and 67 women; mean age \pm standard

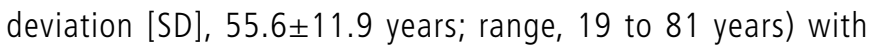
suspected hepatic steatosis, 23 patients $(16.1 \%)$ underwent US examinations as the initial work-up for suspected hepatic steatosis and 120 patients (83.9\%) underwent follow-up US (Table 1). Their mean BMI was $25.7 \pm 3.4 \mathrm{~kg} / \mathrm{m}^{2}$. Seventy-nine patients (79 of $143,55.2 \%$ ) were obese (BMI $\left.\geq 25 \mathrm{~kg} / \mathrm{m}^{2}\right)$. The mean skin-tocapsule distance was $18.8 \pm 3.8 \mathrm{~mm}$ (range, 9 to $34 \mathrm{~mm}$ ). Among 130 patients who underwent laboratory tests on the same day of the US examinations, 51 (39.2\%) showed an abnormal increase of either alanine transaminase or aspartate transaminase. In the visual assessment, 132 patients were assessed as having hepatic steatosis by reviewer 1 and 133 patients by reviewer 2 . The mean ATI of the first session was $0.70 \pm 0.11 \mathrm{~dB} / \mathrm{cm} / \mathrm{MHz}$ (range, 0.46 to 0.99 ) and that of the second session was $0.71 \pm 0.10 \mathrm{~dB} / \mathrm{cm} / \mathrm{MHz}$ (range, 0.45 to 1.00$)$.

\section{Group 2}

A total of 18 healthy volunteers ( 13 women and 5 men; mean age \pm SD, $30.2 \pm 7.3$ years; range, 22 to 57 years) were included. The mean $\mathrm{BMI}$ of all volunteers was $21.2 \pm 2.1 \mathrm{~kg} / \mathrm{m}^{2}$. One person (1 of $18,5.6 \%$ ) was obese $\left(27.8 \mathrm{~kg} / \mathrm{m}^{2}\right)$. The mean skin-to-capsule distance was $14.3 \pm 5.2 \mathrm{~mm}$ (range, 10 to $32 \mathrm{~mm}$ ). Two volunteers (2 of $18,11.1 \%$ ) showed elevated levels of alanine transaminase, with a normal level of aspartate transaminase. In the visual assessment, 16 volunteers ( 16 of $18,88.9 \%$ ) were assessed as having normal hepatic echogenicity without steatosis, another ( 1 of $18,5.6 \%$ ) as having mild steatosis, and the other ( 1 of $18,5.6 \%$ ) as having moderate steatosis by both reviewers 1 and 2 . The mean ATI was $0.52 \pm 0.06 \mathrm{~dB} / \mathrm{cm} / \mathrm{MHz}$ (range, 0.46 to 0.67 ) in the first session, $0.51 \pm 0.08 \mathrm{~dB} / \mathrm{cm} / \mathrm{MHz}$ (range, 0.40 to 0.70 ) in the second session, and $0.52 \pm 0.05 \mathrm{~dB} / \mathrm{cm} / \mathrm{MHz}$ (range, 0.44 to 0.66 ) in the third session.

\section{Technical Aspects of ATI Measurements}

In the first session of group 1, six ATI measurements were obtained

Table 1. Patient characteristics

\begin{tabular}{lcc}
\hline \multicolumn{1}{c}{ Characteristic } & Group 1 $(\mathrm{n}=143)$ & Group 2 ( $=18)$ \\
\hline Age $(\mathrm{yr})$ & $55.6 \pm 11.9$ & $30.2 \pm 7.3$ \\
Sex & & \\
$\quad$ Female & $76(53.1)$ & $5(27.8)$ \\
$\quad$ Male & $67(46.9)$ & $13(72.2)$ \\
BMl (kg/m²) & $25.7 \pm 3.4$ & $21.2 \pm 2.1$ \\
Skin-to-capsule distance (mm) & $18.8 \pm 3.8$ & $14.3 \pm 5.2$ \\
LFT abnormality (\%) & $51 / 130^{\text {a) }}(39.2)$ & $2(11.1)$ \\
Etiology & $46(32.2)$ & $\mathrm{N} / \mathrm{A}$ \\
$\quad$ Chronic hepatitis B & $41(28.7)$ & \\
$\quad$ Chronic hepatitis C & $3(2.1)$ & \\
$\quad$ Biopsy-proven NASH & $2(1.4)$ & $18(100)$ \\
Initial study & $23(16.1)$ & \\
\hline
\end{tabular}

Values are presented as mean \pm standard deviation or number (\%).

BMI, body mass index; LFT, liver function test; NASH, nonalcoholic steatohepatitis; $\mathrm{N} / \mathrm{A}$, not available.

${ }^{a}$ Number of patients with abnormal LFT results among 130 patients who underwent laboratory tests on the same day of the ultrasonography examination. 
in 137 patients $(95.8 \%, 137$ of 143$)$, seven measurements in three patients $(2.1 \%)$, eight measurements in one patient $(0.7 \%)$, nine measurements in one patient $(0.7 \%)$, and 12 measurements in one patient $(0.7 \%)$, in order to obtain six ATI color maps with an $\mathrm{R}^{2}$ value $\geq 0.8$. In the second session, $138(96.5 \%, 138$ of 143$)$ patients underwent six measurements, three $(2.1 \%)$ underwent seven measurements, one $(0.7 \%)$ underwent eight measurements, and one $(0.7 \%)$ underwent nine measurements. In group 2, 16 subjects $(88.9 \%)$ underwent six measurements by three radiologists. Two subjects (11.1\%) underwent seven measurements by one radiologist and six measurements by two radiologists. As the ATI measurements required less than 1 second, less than 1 minute was needed to acquire six ATI color maps in both groups.

\section{Intra-observer and Inter-observer Variability of ATI Measurements}

For the intra-observer reproducibility of ATI, the ICC was 0.929 ( $95 \%$ $\mathrm{Cl}, 0.901$ to 0.949$)$, indicating excellent reliability, and the CoV was $7.1 \%$ (SD, 0.051). The intra-observer reproducibility of each subgroup was shown as ICC and CoV, and CoV was shown not to be influenced by sex, age, BMI, skin-to-capsule distance, or chronic liver disease $(P>0.05)$ (Table 2). For inter-observer variability, the ICC was $0.792(95 \% \mathrm{Cl}, 0.549$ to 0.916$)$, indicating good reliability.

\section{Correlation between ATI Measurements and Hepatic Steatosis Grades}

The $A C$ values in groups 1 and $2(n=161)$ according to the visual grading of hepatic steatosis are presented in Table 3. ATI measurements showed a significant correlation with the visual grade of hepatic steatosis for both reviewer 1 (rho, $0.780 ; \mathrm{P}<0.001$ ) and reviewer 2 (rho, $0.695 ; P<0.001$ ). Fig. 2 shows the distribution of ATI over the visual grades of steatosis. The mean AC values were significantly different across all grades $(P<0.05)$ (Fig. 2$)$. There was no significant difference between the visual grades assigned by the two reviewers $(P=0.131)$, and weighted kappa analysis revealed good agreement (linear weight, kappa, 0.800; standard error, 0.036; $95 \% \mathrm{Cl}, 0.730$ to 0.871 ) between the visual grades assigned by the two reviewers.

\section{Discussion}

In our study, we demonstrated that the test-retest reproducibility of ATI in patients with suspected hepatic steatosis was excellent and the inter-observer agreement in asymptomatic volunteers was good. Furthermore, intra-observer repeatability was not affected by sex, age, BMI, skin-to-capsule distance, or chronic liver disease. Additionally, there were significant correlations between ATI and the
Table 2. Results of subgroup analysis of intra-observer reproducibility in group I

\begin{tabular}{|c|c|c|c|}
\hline Variable & ICC (95\% CI) & $\operatorname{CoV}(\%)$ & P-value ${ }^{a)}$ \\
\hline \multicolumn{4}{|l|}{ Sex } \\
\hline Female $(n=67)$ & $0.910(0.854-0.945)$ & 6.5 & 0.088 \\
\hline Male $(n=76)$ & $0.948(0.915-0.967)$ & 5.3 & \\
\hline \multicolumn{4}{|l|}{ Age (yr) } \\
\hline$<55(n=60)$ & $0.938(0.895-0.963)$ & 5.7 & 0.421 \\
\hline$\geq 55(n=83)$ & $0.921(0.879-0.949)$ & 6.3 & \\
\hline \multicolumn{4}{|l|}{ BMI $\left(\mathrm{kg} / \mathrm{m}^{2}\right)$} \\
\hline$<25(n=64)$ & $0.917(0.863-0.949)$ & 6.1 & 0.993 \\
\hline$\geq 25(n=79)$ & $0.937(0.902-0.960)$ & 6.1 & \\
\hline \multicolumn{4}{|c|}{ Skin-to-capsule distance (mm) } \\
\hline$<19(n=74)$ & $0.918(0.870-0.948)$ & 6.4 & 0.337 \\
\hline$\geq 19(n=69)$ & $0.941(0.904-0.963)$ & 5.7 & \\
\hline \multicolumn{4}{|l|}{ Chronic liver disease } \\
\hline Present $(n=46)$ & $0.929(0.871-0.961)$ & 6.8 & 0.704 \\
\hline Absent $(n=97)$ & $0.928(0.891-0.952)$ & 5.7 & \\
\hline Total & $0.929(0.901-0.949)$ & 7.1 & \\
\hline
\end{tabular}

ICC, intra-class correlation coefficient; $\mathrm{Cl}$, confidence interval; CoV, coefficient of variation; $\mathrm{BMI}$, body mass index.

${ }^{a}$ The coefficient of variation of each subgroup was compared.

Table 3. ATI values according to the visual grade of hepatic steatosis in groups 1 and $2(n=161)$

\begin{tabular}{cccc}
\hline & $\begin{array}{c}\text { Visual grades of } \\
\text { hepatic steatosis }\end{array}$ & ATI & $\begin{array}{c}\text { Correlation } \\
\text { coefficient }^{\text {a) }}\end{array}$ \\
\hline Reviewer 1 & $0(n=27,16.8 \%)$ & $0.54 \pm 0.07(0.46-0.78)$ & Rho $=0.780^{\text {b) }}$ \\
& $1(n=52,32.3 \%)$ & $0.65 \pm 0.07(0.50-0.95)$ & \\
& $2(n=74,46.0 \%)$ & $0.75 \pm 0.07(0.62-0.94)$ & \\
& $3(n=8,5.0 \%)$ & $0.92 \pm 0.06(0.84-1.00)$ & \\
Reviewer 2 & $0(n=26,16.1 \%)$ & $0.55 \pm 0.08(0.46-0.78)$ & Rho $=0.695^{\text {b) }}$ \\
& $1(n=62,38.5 \%)$ & $0.66 \pm 0.08(0.49-0.90)$ & \\
& $2(n=65,40.4 \%)$ & $0.76 \pm 0.09(0.62-0.95)$ & \\
& $3(n=8,5.0 \%)$ & $0.87 \pm 0.09(0.73-1.00)$ & \\
\hline
\end{tabular}

Values are presented as mean \pm standard deviation (range).

ATI, attenuation imaging.

a) Spearman rank correlation. ${ }^{\mathrm{b}} \mathrm{P}<0.001$.

visual assessments of hepatic steatosis performed by each of the two reviewers. Considering these results, we believe that ATI may be of great utility as a screening test in patients with hepatic steatosis.

In recent years, several US-based techniques such as the controlled attenuation parameter (CAP) acquired via FibroScan (Echosens, Paris, France) or the acoustic structure quantification technique have been used to quantify hepatic steatosis [17,26-28]. However, despite the high accuracy of transient elastography $[29,30]$, 


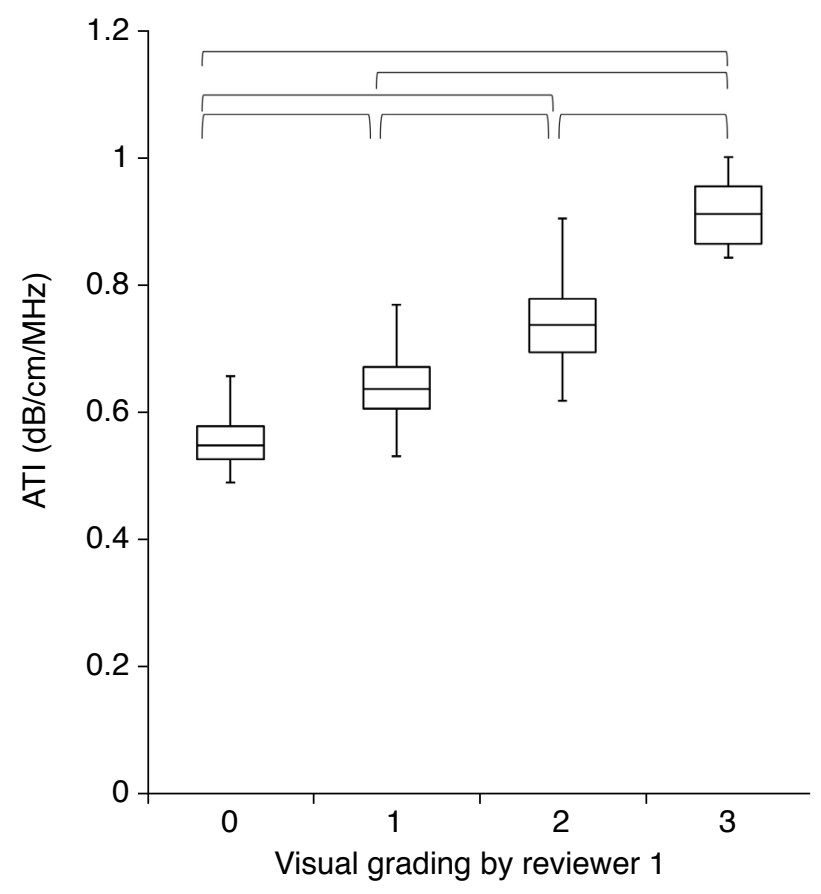

A

Fig. 2. Box-and-whisker plots of the mean attenuation coefficient according to the visually assessed steatosis grade of both (A) reviewer 1 and $(B)$ reviewer 2 .

A, B. Boxes show the median and 25th and 75th percentiles, and whiskers show the minimum and maximum values. Horizontal brackets mean significant differences between each steatosis grade using the Jonckheere-Terpstra and Mann-Whitney U tests. ATI, attenuation imaging.

the diagnostic performance of CAP has been less convincing, especially in obese patients and patients with a heterogeneous distribution of steatosis [31,32]. In contrast, our results showed the maintenance of excellent intra-observer repeatability, irrespective of BMI or skin thickness. This can be explained by the fact that only the reliable areas for measurement were displayed on the ATI map after excluding regions with significant errors in the attenuation calculation; furthermore, the coefficient of determination allowed the operator to confirm the optimal location for ROI placement to improve accuracy. Furthermore, while the unidirectional measurement of CAP may be vulnerable to complex wave patterns such as reflections and refractions [31], ATI presents the adjusted intensity change after removing the system's influence on signal intensity by extracting the focus-dependent beam profile and internal gain control from the observed intensity [21]. Moreover, ATI may be more advantageous as it can be performed with conventional B-mode US, which can help to exclude other causes of liver disease and demonstrate the alteration of hepatic parenchymal echogenicity related with steatosis; additionally, it has a significantly larger area of measurement than CAP [19]. Therefore, we believe that ATI may be utilized as a reliable tool for the evaluation of

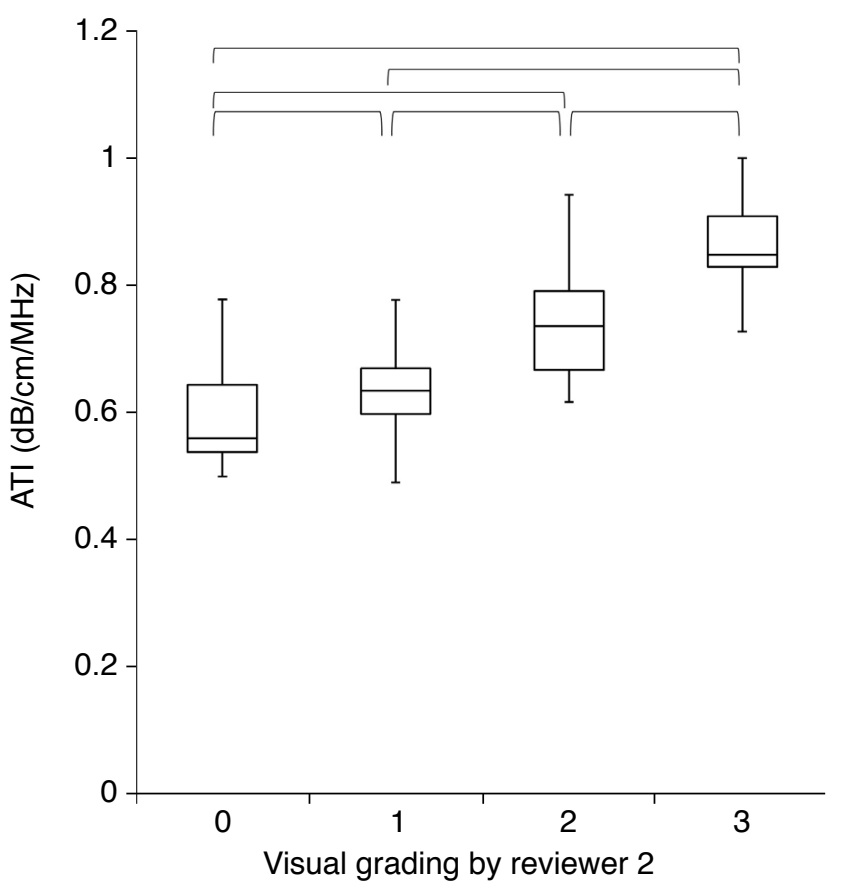

B

\section{(A)}


is $<5 \%$; this discrepancy underscores the need for more robust pathways of risk stratification [27]. Therefore, significant interest has emerged in developing better clinical prediction rules and noninvasive biomarkers to identify NASH in patients with NAFLD [38]. According to a recent practice guideline from the American Association for the Study of Liver Diseases for the diagnosis and management of NAFLD, transient elastography or MR elastography are suggested to be clinically useful tools for identifying advanced fibrosis in patients with NAFLD [38]. Additionally, several major US vendors have developed 2D shear wave elastography (SWE) techniques for hepatic fibrosis staging, and more recently, both 2D ATI and SWE have been equipped in a single clinically available US platform (Canon Medical Systems) [19,39-41]. This promising multiparametric approach using ATI, SWE, and conventional US can provide clinicians with a comprehensive evaluation of parenchymal changes, including quantification of both liver steatosis and stiffness in patients with NAFLD in a single visit, thereby ultimately reducing the need for invasive liver biopsy.

There are several limitations to our study. First, the inter-observer and intra-observer reproducibility of ATI was evaluated in different study populations. However, as this prospective study was performed as an additional part of a routine US examination in the patient population due to funding limitations, it was difficult to perform multiple measurements of ATI with three operators in patients with chronic liver diseases. Instead, inter-observer reproducibility was evaluated in asymptomatic volunteers. Second, we correlated the ATI results using qualitative assessments of B-mode US images rather than a pathologic assessment of hepatic steatosis. However, our study's goal was to evaluate the reproducibility of ATI, rather than to evaluate the diagnostic performance of ATI for hepatic fat quantification. Thus, further prospective studies are warranted to evaluate the diagnostic accuracy of ATI in hepatic fat quantification using pathologic assessment or MR-proton density fat fraction as a reference standard in patients who underwent liver transplantation. Third, the test-retest reproducibility of ATI was tested on the same day with a 2-minute interval, although positional changes were made after the first set of measurements. Furthermore, the fact that a radiologist who was aware of the results of the ATI measurements of the first session performed the second session of ATI measurements might have had an effect on the intra-observer reproducibility. However, as we included all ATI measurements with $R^{2}$ value $>0.8$, the impact on intra-observer reproducibility is unlikely to have been excessive. Since ATI could be used for longitudinal follow-up of patients with NAFLD or patients with steatosis and chronic liver diseases after modification of lifestyle and diet control, test-retest reproducibility on different days would have been more desirable.
In conclusion, ATI demonstrated excellent intra-observer reproducibility and good inter-observer reproducibility in the assessment of hepatic steatosis.

ORCID: Jeongin Yoo: https://orcid.org/0000-0002-3267-2544; Jeong Min Lee: https://orcid.org/0000-0003-0561-8777; ljin Jo0: https://orcid.org/0000-00021341-4072; Dong Ho Lee: https://orcid.org/0000-0001-8983-851X; Jeong Hee Yoon: https://orcid.org/0000-0002-9925-9973; Hyo-Jin Kang: https://orcid.org/0000-0002$6771-2112$

\section{Author Contributions}

Conceptualization: Lee JM. Data acquisition: Lee JM, Kang HJ, Ahn SJ. Data analysis or interpretation: Yoo J, Lee JM. Drafting of the manuscript: Yoo J. Critical revision of the manuscript: Lee JM, Joo I, Lee $\mathrm{DH}$, Yoon JH. Approval of the final version of the manuscript: all authors.

\section{Conflict of Interest}

This work was supported by Canon Medial Systems Corporation (Tochigi, Japan). The funding source had no involvement in any phase of this work.

\section{Acknowledgments}

We thank Chris Woo, B.A. for his assistance in editing the manuscript.

\section{References}

1. Ress C, Kaser S. Mechanisms of intrahepatic triglyceride accumulation. World J Gastroenterol 2016;22:1664-1673.

2. Bedogni G, Bellentani S, Miglioli L, Masutti F, Passalacqua M, Castiglione A, et al. The Fatty Liver Index: a simple and accurate predictor of hepatic steatosis in the general population. BMC Gastroenterol 2006;6:33.

3. Younossi ZM, Koenig AB, Abdelatif D, Fazel Y, Henry L, Wymer M. Global epidemiology of nonalcoholic fatty liver disease: metaanalytic assessment of prevalence, incidence, and outcomes. Hepatology 2016;64:73-84.

4. Rinella ME. Nonalcoholic fatty liver disease: a systematic review. JAMA 2015;313:2263-2273.

5. Baclig MO, Reyes KG, Liles VR, Mapua CA, Dimamay MP, GopezCervantes J. Hepatic steatosis in chronic hepatitis B: a study of metabolic and genetic factors. Int J Mol Epidemiol Genet 2018:9:13-19.

6. Yoon EJ, Hu KQ. Hepatitis C virus (HCV) infection and hepatic steatosis. Int J Med Sci 2006;3:53-56.

7. Seto WK, Hui RW, Mak LY, Fung J, Cheung KS, Liu KS, et al. Association between hepatic steatosis, measured by vontrolled 
attenuation parameter, and fibrosis burden in chronic hepatitis B. Clin Gastroenterol Hepatol 2018;16:575-583.

8. Hui RW, Seto WK, Cheung KS, Mak LY, Liu KS, Fung J, et al. Inverse relationship between hepatic steatosis and hepatitis $B$ viremia: results of a large case-control study. J Viral Hepat 2018;25:97-104.

9. Romero-Gomez M, Cortez-Pinto H. Detecting liver fat from viscoelasticity: how good is CAP in clinical practice? The need for universal cut-offs. J Hepatol 2017;66:886-887.

10. Castera L. Steatosis, insulin resistance and fibrosis progression in chronic hepatitis C. Minerva Gastroenterol Dietol 2006;52:125134.

11. Di Lascio N, Avigo C, Salvati A, Martini N, Ragucci M, Monti S, et al. Steato-Score: non-invasive quantitative assessment of liver fat by ultrasound imaging. Ultrasound Med Biol 2018;44:1585-1596.

12. Sumida $Y$, Nakajima $A$, Itoh $Y$. Limitations of liver biopsy and noninvasive diagnostic tests for the diagnosis of nonalcoholic fatty liver disease/nonalcoholic steatohepatitis. World J Gastroenterol 2014;20:475-485.

13. Ratziu V, Charlotte F, Heurtier A, Gombert S, Giral P, Bruckert E, et al. Sampling variability of liver biopsy in nonalcoholic fatty liver disease. Gastroenterology 2005;128:1898-1906.

14. Dulai PS, Sirlin CB, Loomba R. MRI and MRE for non-invasive quantitative assessment of hepatic steatosis and fibrosis in NAFLD and NASH: Clinical trials to clinical practice. J Hepatol 2016;65:1006-1016.

15. Yoon JH, Lee JM, Joo I, Lee ES, Sohn JY, Jang SK, et al. Hepatic fibrosis: prospective comparison of MR elastography and US shearwave elastography for evaluation. Radiology 2014;273:772-782.

16. Crossan C, Tsochatzis EA, Longworth L, Gurusamy K, Davidson B, Rodriguez-Peralvarez $M$, et al. Cost-effectiveness of non-invasive methods for assessment and monitoring of liver fibrosis and cirrhosis in patients with chronic liver disease: systematic review and economic evaluation. Health Technol Assess 2015;19:1-409.

17. Son JY, Lee JY, Yi NJ, Lee KW, Suh KS, Kim KG, et al. Hepatic steatosis: assessment with acoustic structure quantification of US imaging. Radiology 2016;278:257-264.

18. Paige JS, Bernstein GS, Heba E, Costa EA, Fereirra M, Wolfson $T$, et al. A pilot comparative study of quantitative ultrasound, conventional ultrasound, and MRI for predicting histologydetermined steatosis grade in adult nonalcoholic fatty liver disease. AJR Am J Roentgenol 2017;208:W168-W177.

19. Kanayama Y, Kamiyama N, Maruyama K, Sumino Y. Real-time ultrasound attenuation imaging of diffuse fatty liver disease. Ultrasound Med Biol 2013;39:692-705.

20. Han A, Andre MP, Deiranieh L, Housman E, Erdman JW Jr, Loomba R, et al. Repeatability and reproducibility of the ultrasonic attenuation coefficient and backscatter coefficient measured in the right lobe of the liver in adults with known or suspected nonalcoholic fatty liver disease. J Ultrasound Med 2018;37:1913-1927.
21. Jirik $R$, Taxt $T$, Jan J. Ultrasound attenuation imaging. J Electr Eng 2004;55:180-187.

22. Sasso $M$, Beaugrand $M$, de Ledinghen $V$, Douvin $C$, Marcellin $P$, Poupon $R$, et al. Controlled attenuation parameter (CAP): a novel VCTE guided ultrasonic attenuation measurement for the evaluation of hepatic steatosis: preliminary study and validation in a cohort of patients with chronic liver disease from various causes. Ultrasound Med Biol 2010;36:1825-1835.

23. Ballestri S, Lonardo A, Romagnoli D, Carulli L, Losi L, Day CP, et al. Ultrasonographic fatty liver indicator, a novel score which rules out $\mathrm{NASH}$ and is correlated with metabolic parameters in NAFLD. Liver Int 2012;32:1242-1252.

24. Saadeh S, Younossi ZM, Remer EM, Gramlich T, Ong JP, Hurley M, et al. The utility of radiological imaging in nonalcoholic fatty liver disease. Gastroenterology 2002;123:745-750.

25. Portney LG, Watkins MP. Foundations of clinical research: applications to practice. 2nd ed. Upper Saddle River, NJ: Prentice Hall Health, 2000.

26. Lee DH, Lee JY, Lee KB, Han JK. Evaluation of hepatic steatosis by using acoustic structure quantification US in a rat model: comparison with pathologic examination and MR spectroscopy. Radiology 2017;285:445-453.

27. Tsochatzis EA, Newsome PN. Non-alcoholic fatty liver disease and the interface between primary and secondary care. Lancet Gastroenterol Hepatol 2018;3:509-517.

28. Lee HW, Park SY, Kim SU, Jang JY, Park H, Kim JK, et al. Discrimination of nonalcoholic steatohepatitis using transient elastography in patients with nonalcoholic fatty liver disease. PLoS One 2016;11:e0157358.

29. Fraquelli M, Rigamonti C, Casazza G, Conte D, Donato MF, Ronchi $\mathrm{G}$, et al. Reproducibility of transient elastography in the evaluation of liver fibrosis in patients with chronic liver disease. Gut 2007; 56:968-973.

30. Tsochatzis EA, Gurusamy KS, Ntaoula S, Cholongitas E, Davidson $B R$, Burroughs AK. Elastography for the diagnosis of severity of fibrosis in chronic liver disease: a meta-analysis of diagnostic accuracy. J Hepatol 2011;54:650-659.

31. Imajo K, Kessoku T, Honda Y, Tomeno W, Ogawa Y, Mawatari $H$, et al. Magnetic resonance imaging more accurately classifies steatosis and fibrosis in patients with nonalcoholic fatty liver disease than transient elastography. Gastroenterology 2016;150:626-637.

32. Petta S, Wong VW, Camma C, Hiriart JB, Wong GL, Marra F, et al. Improved noninvasive prediction of liver fibrosis by liver stiffness measurement in patients with nonalcoholic fatty liver disease accounting for controlled attenuation parameter values. Hepatology 2017;65:1145-1155.

33. McPherson S, Jonsson JR, Cowin GJ, O'Rourke P, Clouston AD, Volp $A$, et al. Magnetic resonance imaging and spectroscopy accurately estimate the severity of steatosis provided the stage of fibrosis is 
considered. J Hepatol 2009;51:389-397.

34. Kalra N, Duseja A, Das A, Dhiman RK, Virmani V, Chawla Y, et al. Chemical shift magnetic resonance imaging is helpful in detecting hepatic steatosis but not fibrosis in patients with nonalcoholic fatty liver disease (NAFLD). Ann Hepatol 2009;8:21-25.

35. Friedrich-Rust M, Muller C, Winckler A, Kriener S, Herrmann E, Holtmeier J, et al. Assessment of liver fibrosis and steatosis in PBC with FibroScan, MRI, MR-spectroscopy, and serum markers. J Clin Gastroenterol 2010;44:58-65.

36. Idilman IS, Aniktar H, Idilman R, Kabacam G, Savas B, Elhan A, et al. Hepatic steatosis: quantification by proton density fat fraction with MR imaging versus liver biopsy. Radiology 2013;267:767-775.

37. Cholet F, Nousbaum JB, Richecoeur M, Oger E, Cauvin JM, Lagarde $\mathrm{N}$, et al. Factors associated with liver steatosis and fibrosis in chronic hepatitis C patients. Gastroenterol Clin Biol 2004;28:272278.

38. Chalasani N, Younossi Z, Lavine JE, Diehl AM, Brunt EM, Cusi K, et al. The diagnosis and management of non-alcoholic fatty liver disease: practice Guideline by the American Association for the Study of Liver Diseases, American College of Gastroenterology, and the American Gastroenterological Association. Hepatology 2012;55:2005-2023.

39. Sigrist RM, Liau J, Kaffas AE, Chammas MC, Willmann JK. Ultrasound elastography: review of techniques and clinical applications. Theranostics 2017;7:1303-1329.

40. Maruyama H, Kobayashi K, Kiyono S, Sekimoto T, Kanda T, Yokosuka O. Two-dimensional shear wave elastography with propagationbased reliability assessment for grading hepatic fibrosis and portal hypertension. J Hepatobiliary Pancreat Sci 2016;23:595-602.

41. Lee ES, Lee JB, Park HR, Yoo J, Choi Jl, Lee HW, et al. Shear Wave Liver Elastography with a propagation map: diagnostic performance and Inter-observer correlation for hepatic fibrosis in chronic hepatitis. Ultrasound Med Biol 2017;43:1355-1363. 